\title{
Molecular Interactions between Erythrocytes and the Endocrine System
}

\author{
Charalampos PAPADOPOULOS, Ioannis TENTES, \\ Konstantinos ANAGNOSTOPOULOS
}

Laboratory of Biochemistry, School of Medicine, Democritus University of Thrace, Alexandroupolis, Greece

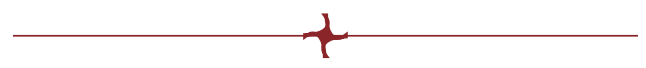

\begin{abstract}
- ABSTRACT
Hormones are secreted by the endocrine glands and reach their targets after circulating in the blood. Many studies have documented that erythrocytes can bind hormones, and possible interactions have been reported. Erythrocytes are responsive to signaling initiated after binding of epinephrine, norepinephrine, estrogen, progesterone, thyroid hormones, parathyroid hormone, and angiotensin. Signaling results in regulation of cellular metabolism and membrane fluidity. In addition, erythrocytes are circulating pools for dopamine, thyroid hormones, cortisol, and aldosterone. Erythrocyte function and structure are regulated by endocrine signals, while erythrocytes are important constituents for the transport of hormones in the body.
\end{abstract}

Keywords: erythrocyte, hormones, endocrine system, nitric oxide, metabolism.

\section{INTRODUCTION}

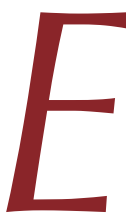
rythrocytes have recently emerged as important contributors to the regulation of immunity $(1,2)$. Furthermore, we have recently described the current literature that indicates that red blood cells constituted a dynamic pool of cholesterol and signaling lipids (2). In addition, erythrocytes comprise an important pool of the bioactive gas, nitric oxide (NO) (3). However, evidence points also toward the direction of an erythrocyte-hormone interaction and/or transport (Figure 1).
Thus, in this review we have tried to summarize the current knowledge regarding the molecular interactions between erythrocytes and hormones in mammals, with a particular interest in humans. However, as the interaction of erythrocytes with insulin and non-neuronal acetylcholine has already been summarized in other excellent reviews $(4,5)$, it will not be discussed here. In addition, the interaction of erythrocytes with lipid hormones has recently been described as a part of metabolic interactions, and will therefore not be described (2).

\footnotetext{
Address for correspondence:

Konstantinos Anagnostopoulos, $\mathrm{PhD}$

Laboratory of Biochemistry, School of Medicine, Democritus University of Thrace, 68100 Alexandroupolis, Greece

Tel./Fax: +30 2551030502

Email:kanagnos@med.duth.gr,kanagno@gmail.com
}

Article received on the $1^{\text {st }}$ of April 2021 and accepted for publication on the $3^{\text {rd }}$ of September 2021 


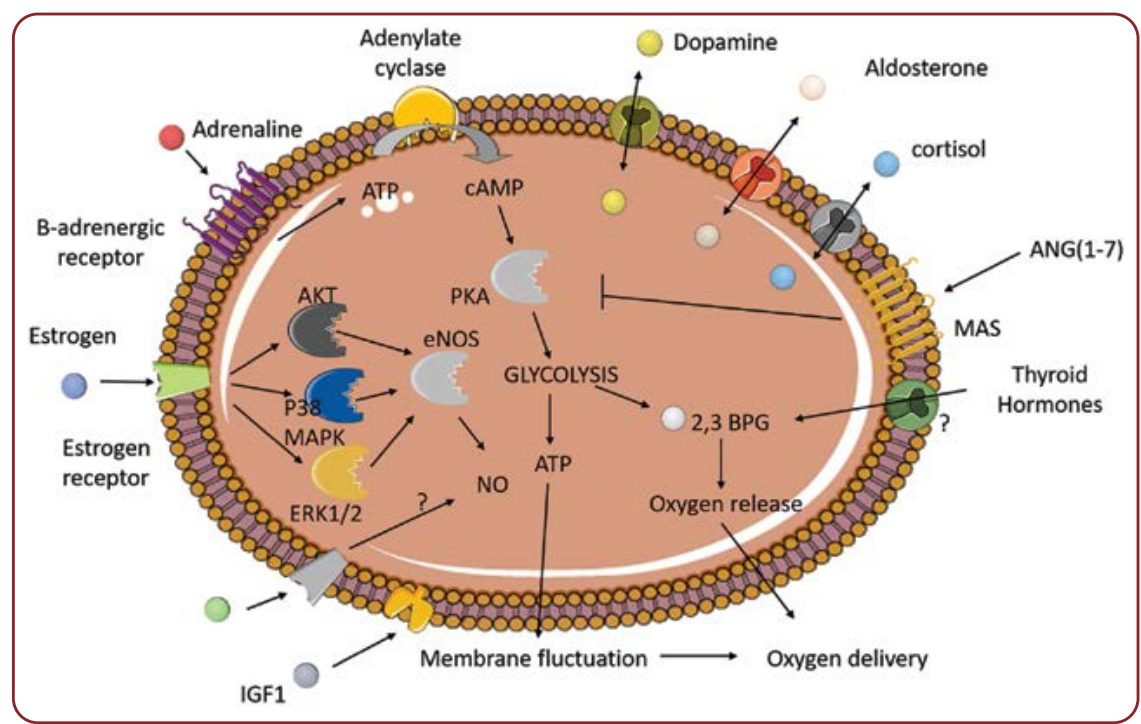

FIGURE 1. Molecular interactions between red blood cells and hormones.

AKT: Rasrelated C3 botulinum toxin alpha serine/threonine-kinase ANG(1-7): angiotensin 1-7; ATP: adenosine triphosphate; 2,3 BPG: 2,3 biphosphoglycerate; cAMP: cyclin adenosine monophosphate; eNOS: endothelial nitric oxide synthase; ERK: extracellular signal-regulated kinase; IGF1: insulin-like growth factor 1; MAPK: mitogen activated protein kinase; NO: nitric oxide; PKA: protein kinase A

\section{Epinephrine and norepinephrine}

Tsukamoto and Sonenberg (6) provided the first evidence that the erythrocyte membrane possessed a functional beta-adrenergic receptor. In their experiments, both epinephrine and norepinephrine resulted in an increased activity of the cAMP-activated protein kinase of red blood cells. This mechanism was inhibited when using an antagonist for the beta-adrenergic receptor. Later, this idea has been also supported by the results of Horga et al (7), who showed that incubation of erythrocyte membranes with isoproterenol, an agonist for the beta-adrenergic receptor, led to the activation of adenylic cyclase. This adrenaline $\rightarrow$ beta-adrenergic receptor $\rightarrow$ adenylic cyclase $\rightarrow$ cAMP pathway was implicated in the increase of oxygen supply, which was induced by high-fat diet-induced hypoxia (8). In that study, activation of beta-adrenergic receptors induced an increase in CAMP, glycolysis, and ATP production. This pathway was correlated with an increased oxygen supply, thus aiding with the hypoxic environment. The precise mechanism for this function is not known. Nevertheless, researchers had previously shown that ATP production, induced by adrenergic signaling in erythrocytes, results in increased membrane fluctuation, a mechanism facilitating erythrocyte passage and oxygen delivery (9). However, this pathway may present resistance. Increased epinephrine levels in the blood of horses after maximal exercise resulted in a decrease of the beta-adrenergic receptors of red blood cells (10).

However, it is speculated that the mode of induction of hypoxia and subsequent adrenergic signaling in erythrocytes could lead to different intracellular pathways. Odje and Ramsey (11) showed that epinephrine resulted in augmentation of 2,3 diphosphoglycerate. Interestingly, 2,3 diphosphoglycerate, an intermediate molecule of glycolysis, also increases oxygen release since it is an allosteric modulator of hemoglobin (12). However, this pathway was found to be initiated by the production of sphingosine- 1 phosphate.

\section{Dopamine}

Red blood cells have been proposed as transporters of catecholamines and especially dopamine. Azoui et al (13) identified a choline exchanger as a potent transporter of dopamine in the erythrocyte membrane. In addition, experimental work, both in rats and in vitro, proved that dopamine inserted the erythrocyte mem- 
brane. Interestingly, the erythrocyte storage capacity for dopamine seems to be saturable (14).

\section{Estrogen}

Functional estrogen receptors (Era and $\mathrm{ERb}$ ) were found in erythrocytes (15). In fact, the same study found that estrogen receptors were mainly located in the cytosol, and estrogen triggered their localization to the plasma membrane. Activation of these receptors leads to activation of AKT, ERK1/2, and p38. Additionally, eNOS activation by estrogen was reported in both genders, but women presented higher basal levels due to their exposure to higher levels of estrogen.

\section{Progesterone}

Progesterone has been found to regulate the fluidity of red blood cells (16). Interestingly, the addition of an inhibitor of NO synthase augmented this effect. These results may imply that erythrocytes also possess progesterone receptors in their membranes.

\section{Insulin-like growth factor-1}

Moris et al (17) report that insulin-like growth factor 1 (IGF-1) binds to a receptor of erythrocytes. In addition, there was found an increased affinity for binding to erythrocytes derived from prepubertal children in comparison with erythrocytes from adults. This was not ascribed to the number of receptors in the erythrocyte membranes in the two groups. These results may imply that specific intra-erythrocyte signaling induced by IGF-1 is taking place during puberty.

\section{Thyroid hormones}

Snyder and Reddy (18) showed that thyroid hormones upregulated the levels of 2,3 biphosphoglyceric in erythrocytes, thus implying a possible connection with the regulation of oxygen release from hemoglobin. In addition, red blood cells were deemed possible circulation pools of the thyroid hormones T3 and T4, since erythrocytes were found to trap and release both hormones at similar rates (19).

\section{Parathyroid hormone}

The parathyroid hormone binds to and induces calcium entry via calcium ATPase and subsequent cytoskeletal organization disruption (20).

\section{Angiotensin}

Saraiva et al (21) showed that angiotensin II was converted to angiotensin (1-7) and angiotensin (IV) by human erythrocytes. Next, these molecules lead to decreased Gs protein activation, mainly through the MAS receptor and partially through the angiotensin receptor 2, and subsequently, to decreased protein kinase A (PKA) activity.

\section{Cortisol}

Erythrocytes can serve as transporters of cortisol in the blood through binding and releasing with high dissociation rates (22). In addition, this transport is possibly temperature-dependent (23).

\section{Aldosterone}

Similarly, erythrocytes bind and transport aldosterone in the blood temperature dependently (24).

\section{CONCLUSION}

Erythrocytes constitute both targets and trans- porters of various hormones in the circulation. Studies show that hormonal signals can regulate oxygen supply. In addition, erythrocytes are important, yet underappreciated transporters of hormones. We propose that red blood cells are circulating pools of hormones, cytokines, bioactive lipids, cholesterol, and damage-associated molecular patterns. Finally, oxygen release is a dynamic biochemical event regulated by external signals, and whole-body and intra-erythrocyte metabolism. Thus, erythrocytes could lead to the discovery of therapeutic targets for a disease where hypoxia and/or hormone circulation takes place.

Conflicts of interest: none declared.

Financial support: The research work was supported by the Hellenic Foundation for Research and Innovation (HFRI) under the HFRI PhD Fellowship grant (Fellowship Number: 1343). 


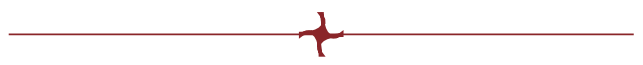

\section{R}

1. Anderson HL, Brodsky IE, Mangalmurti NS. The Evolving Erythrocyte: Red Blood Cells as Modulators of Innate Immunity. I Immunol 2018;201:1343-1351.

2. Papadopoulos $C$, Panopoulou $M$, Anagnostopoulos K, et al. Immune and Metabolic Interactions of Human Erythrocytes: A Molecular Perspective. Endocrine, Metab Immune Disord - Drug Targets 2020;20.

3. Simmonds MJ, Detterich JA, Connes P. Nitric oxide, vasodilation and the red blood cell. In: Biorheology, IOS Press, 2014 pp 121-134.

4. Gambhir KK, Agarwal VR. Red blood cell insulin receptors in health and disease. In: Biochemical Medicine and Metabolic Biology, Academic Press, 1991, vol. 45, pp 133-153.

5. De Almeida JPL, Saldanha C. Nonneuronal cholinergic system in human erythrocytes: Biological role and clinical relevance [Internet]. J Membr Biol 2010;234:227-234.

6. Tsukamoto $T$, Sonenberg $M$. Catecholamine regulation of human erythrocyte membrane protein kinase. J Clin Invest 1979;64:534-540.

7. Horga JF, Gisbert J, De Agustín JC, et al. A beta-2-adrenergic receptor activates adenylate cyclase in human erythrocyte membranes at physiological calcium plasma concentrations. Blood Cells, Mol Dis 2000;26:223-228.

8. Kim ER, Fan S, Akhmedov D, et al. Red blood cell $\beta$-adrenergic receptors contribute to diet-induced energy expenditure by increasing $\mathrm{O}_{2}$ supply. JCI Insight 2017;2:e93367.

9. Tuvia S, Moses A, Gulayev N, et al. $\beta$-adrenergic agonists regulate cell membrane fluctuations of human erythrocytes.

J Physiol 1999;516:781-792.

10. González O, González E, Sánchez $C$, et al. Effect of exercise on erythrocyte $\beta$-adrenergic receptors and plasma concentrations of catecholamines and thyroid hormones in Thoroughbred horses. Equine Vet J 1998;30:72-78.

11. Odje OE, Ramsey JM. Effect of adrenaline on the response of erythrocyte 2,3-diphosphoglycerate in rabbits in vivo. Gen Pharmacol 1996;27:651-653.

12. Sun $K$, Zhang $Y$, $D^{\prime}$ Alessandro A, et al. Sphingosine-1-phosphate promotes erythrocyte glycolysis and oxygen release for adaptation to high-altitude hypoxia. Nat Commun 2016;7:12087.

13. Azoui R, Cuche JL, Renaud JF, et al. A dopamine transporter in human erythrocytes: Modulation by insulin. Exp Physiol 1996;81:421-434.

14. Azoui R, Schneider J, Dong WX, et al. Red blood cells participate in the metabolic clearance of catecholamines in the rat. Life Sci 1997;60:357-367.

15. Vona R, Gambardella L, Ortona E, et al. Functional estrogen receptors of red blood cells. Do they influence intracellular signaling? Cell Physiol Biochem 2019;53:186-199.

16. Tsuda K, Kinoshita Y, Nishio I. Synergistic role of progesterone and nitric oxide in the regulation of membrane fluidity of erythrocytes in humans: An electron paramagnetic resonance investigation. Am J Hypertens 2002;15:702-708.

17. Morris AH, Joyce JL, Reiter EO.
Increased Insulin-Like Growth Factor I Binding to Red Blood Cells of Normal Prepubertal Children1.

Pediatr Res 1989;25:409-413.

18. Snyder LM, Reddy WJ. Mechanism of action of thyroid hormones on erythrocyte 2,3-diphosphoglyceric acid synthesis.

J Clin Invest 1970;49:1993-1998.

19. Osty J, Valensi $P$, Samson $M$, et al. Transport of thyroid hormones by human erythrocytes: Kinetic characterization in adults and newborns. J Clin Endocrinol Metab 1990;71:1589-1595.

20. Bogin E, Massry SG, Levi J, et al. Effect of parathyroid hormone on osmotic fragility of human erythrocytes. J Clin Invest 1982;69:1017-1025.

21. Saraiva VB, de Souza Silva L, Ferreira-DaSilva CT, et al. Impairment of the Plasmodium falciparum erythrocytic cycle induced by angiotensin peptides. PLoS One 2011;6:17174.

22. Hiramatsu R, Nisula BC. Erythrocyte-Associated Cortisol: Measurement, Kinetics of Dissociation, and Potential Physiological Significance. I Clin Endocrinol Metab 1987;64:1224-1232.

23. Lentjes EGWM, Romijn FHTPM. Temperature-dependent cortisol distribution among the blood compartments in man. J Clin Endocrinol Metab 1999;84:682-687.

24. Chavarri M, Luetscher JA, Dowdy AJ, et al. The effects of temperature and plasma cortisol on distribution of aldosterone between plasma and red blood cells: Influence on metabolic clearance rate and on hepatic and renal extraction of aldosterone.

J Clin Endocrinol Metab 1977;44:752-759. 